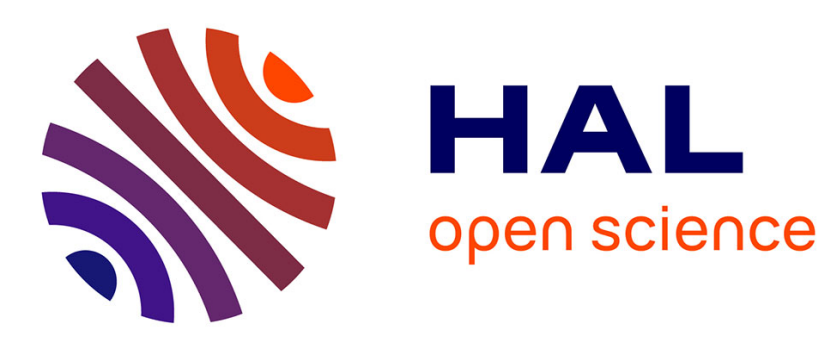

\title{
Design patterns for environments in multi-agent simulations
}

Philippe Mathieu, Sébastien Picault, Yann Secq

\section{To cite this version:}

Philippe Mathieu, Sébastien Picault, Yann Secq. Design patterns for environments in multi-agent simulations. Principles and Practice of Multi-Agent Systems, PRIMA 2015, 18th International Conference, Oct 2015, Bertinoro, Italy. pp.678-686, 10.1007/978-3-319-25524-8_51 . hal-01248799

\section{HAL Id: hal-01248799 \\ https://hal.inria.fr/hal-01248799}

Submitted on 15 Feb 2016

HAL is a multi-disciplinary open access archive for the deposit and dissemination of scientific research documents, whether they are published or not. The documents may come from teaching and research institutions in France or abroad, or from public or private research centers.
L'archive ouverte pluridisciplinaire HAL, est destinée au dépôt et à la diffusion de documents scientifiques de niveau recherche, publiés ou non, émanant des établissements d'enseignement et de recherche français ou étrangers, des laboratoires publics ou privés. 


\title{
Design patterns for environments in multi-agent simulations
}

\author{
Philippe Mathieu, Sébastien Picault, and Yann Secq \\ SMAC team, CRIStAL UMR CNRS 9189 \\ University of Lille - Science and Technology \\ 59655 Villeneuve d'Ascq cedex, France \\ \{philippe.mathieu, sebastie.picault, yann.secq\}@univ-lille1.fr \\ http://cristal.univ-lille.fr/SMAC
}

\begin{abstract}
In the field of multi-agent based simulation (MABS), the concept of environment is omnipresent, though poorly defined. We argue here that depending on the modeling of space and of relations between agents, only a few efficient implementations can be set up. We aim at formalizing the core functions of environments, so as to highlight the computational answers to possible modeling choices. This unifying approach leads to the identification of four paradigmatic Design Patterns, associated with specific atomic environments, which can be composed in order to tackle complex situations.
\end{abstract}

Keywords: Environments; Modeling; Parsimony; Engineering; Design Patterns

\section{Introduction}

In this paper, we focus on the definition and on the implementation of the concept of "environment" in MABS. It is generally admitted that agents act "in an environment" $[1,2]$, and most of the time "environments are simply assumed as given" [3]. Indeed everybody is supposed to know what an environment is, but this implicit reference to common sense makes the environment the neglected concept in MABS design. Hence, the term itself refers to heterogeneous realities, from an abstract description of an "habitat" (in an ecological sense) where the agents "live", to the hardware/software execution infrastructure of a platform [4] and interoperability issues [5,6]. Some exceptions are found in Agent-Based Simulation, where the issue is crucial $[7,8]$. Several criteria for characterizing environments have been proposed by [2, ch. 2] (e.g. accessibility, determinism, static vs. dynamic, continuous vs. discrete), yet a close reading shows that they are not exactly environment features, but rather peception, cognition and action capabilities of the agents that are situated in it.

In contrast, [4] addresses crucial issues such as eliciting the link between agents and environments, the need for a taxonomy of environments, or the essential role of topological relations. Indeed, in order to act each agent needs to 
know on which other agents it can perform actions (including communication). As the agent has only a limited (local) perception, it can (must) interact only with its neighbors. Thus, the main issue is: how does an agent determine its neighbors? Essentially, the environment ought to be an answer to this question.

Our goal in this paper is to search for a minimal, formal definition of what the term "environment" can mean in a MABS, and to show how to reduce the diversity of environments found in existing MABS to a few combinable patterns, as suggested by [9]. We argue that the choice of appropriate techniques for modeling environments only depends on a very limited number of criteria, as in the classical Design Patterns used in software engineering [10].

In the following, each entity of the simulation model is represented by an agent as suggested in [11,12]. We also assume discrete time, so that, at each time step, the MABS contains a set of agents, denoted by $\mathcal{A}_{t}$. At this stage we do not assume any special hypothesis regarding the capabilities of action, perception or cognition of those entities. From the environment indeed, "objects", "artifacts" or "agents" are undiscernible (proactivity being an internal feature). Obviously though, everything is not meant to be reified by agents: depending on the analysis level required in the model, there is a granularity level, beyond which would-be entities are modeled by an aggregated information (see $\S 2.2$ ).

In the next section, we propose a minimal abstract definition of a MABS environment; then, we describe four patterns induced by modeling and implementation choices; finally, we explain how those patterns may be composed for building usual MABS environments.

\section{Formalizing the concept of environment}

We advocate that there are two main roles devoted to the environment in a MABS: first, to place agents so as to define neighborhood and accessibility relationships; second, to provide spatial information, which reflects underlying levels that would not be reified through agents.

\subsection{First purpose: placing agents}

We suppose a set $E$ composed of "places" which can host agents, and can be arbitrarily chosen w.r.t the nature of the world to be modeled. To determine accessibility relations from one place to the others, we also suppose a distance function $\mathbf{d}: \mathbf{E} \times \mathbf{E} \rightarrow \mathbb{R}^{+} \cup\{+\infty\}$, so that $(E, d)$ is a metric space in which the distance induces a topology. This minimalistic description covers many situations, e.g. the usual 3D space $\left(E=\mathbb{R}^{3}\right)$ with the euclidean distance; a $2 \mathrm{D}$ grid with the Moore neighborhood ( $E=\mathbb{Z}^{2}, d$ being the Chebychef distance); a continuous 2D "toric" space of width $w$ and height $h$ (quotient space $E=\mathbb{R}^{2} /(w \mathbb{Z} \times h \mathbb{Z})$ ); an acquaintance network (graph, i.e. a set $E$ of nodes, endowed with the geodesic distance); etc. "Non situated" agent corresponds to a singleton $E=\{p\}$, endowed with the trivial distance $\forall x, y d(x, y)=0$. Generally only a subset $\mathcal{E} \subset E$ is used in the simulation. 
Since the first purpose of the environment is to locate agents, $\mathcal{E}$ must be endowed with a function designed to provide the agents with a position at each time step: pos $_{t}: \mathcal{A}_{t} \rightarrow \mathcal{E}$. Then, an environment can be defined as a subset $\mathcal{E}$ of a metric space $(E, d)$ endowed with a family of functions $\left(\text { pos }_{t}\right)_{t \in\left[0, T_{\max }\right]}$ which give the successive positions of agents within $\mathcal{E}$. Those functions can often be specified only in extension; besides, each agent $a$ is responsible for handling the value of $\operatorname{pos}_{t}(a)$ (e.g. as an attribute). Moreover, the transition between $\operatorname{pos}_{t}$ and $p s_{t+1}$ is itself the consequence of the behavior of each agent. Nevertheless, this function-based description also covers cases where positions can be specified in intension (e.g. trajectories following physical laws). We also define the reciprocal function of the position, i.e. the content of a point of the environment, as follows: $\forall p \in \mathcal{E}, \boldsymbol{c o n t}_{t}(p)=\left\{a \in \mathcal{A}_{t} \mid \operatorname{pos}_{t}(a)=p\right\}$.

From this formalization, we claim that two features can discriminate between several kinds of environments and their possible implementation: first, the nature of the metric space $(E, d)$ (continuous, lattice, graph, singleton...), and second, the decision (or need) to provide explicitly those functions, especially cont ${ }_{t}$. Indeed, the behavior of agents crucially depends on their neighborhood function, devoted to identifying their neighbors, i.e. with whom they can interact, namely $\nu_{t}: \mathcal{A}_{t} \rightarrow \wp\left(\mathcal{A}_{t}\right)$. Among an infinite variety of possible functions, a simple example is the topological ball centered on the position of $a$ and of radius $r$ : $\nu_{t}^{r}(a)=\left\{a^{\prime} \in \mathcal{A}_{t} \backslash\{a\} \mid d\left(\operatorname{pos}_{t}(a), \operatorname{pos}_{t}\left(a^{\prime}\right)\right) \leq r\right\}$. The computational cost of computing a neighborhood may require a time/space compromise between algorithmic efficiency and data structures. Thus a given neighborhood function may be implemented through several forms, as explained in section 3 .

\subsection{Second purpose: providing information}

Depending on the modeling scale, some underlying levels may require a macroscopic approximation. For instance, pheromones, as entities (molecules) should be represented by agents (endowed with physico-chemical behaviors: brownian motion, evaporation, degradation). But, in practice, they are usually aggregated numerically (like the concentration in chemistry) and mapped to a discretized space (the cells of a grid), in the mere intend of reducing the computational cost of handling a large number of entities, but at the expense of approximating diffusion phenomena. The MAS literature does not agree on any convention for naming such a purely informational representation. The concept of field, already used in reactive navigation or for pheromones (Potential Fields [13] and Mean Fields [14]) seems a convenient way of modeling any kind of "information" afforded by the environment (e.g. gravitation forces, temperature, obstacles) and not reified by agents. A field is thus a function which maps points of $\mathcal{E}$ to any set of information pieces $\mathcal{I}$; e.g. the pheromone level in grid cells would be: phero $_{t}: \mathbb{Z}^{2} \rightarrow \mathbb{R}^{+}$, defined in extension and changing at each time step through a classical diffusion-evaporation algorithm [15]. A field can sometimes be defined in intension, e.g. the gravity field in the usual $3 \mathrm{D}$ space $\boldsymbol{g}: \mathbb{R}^{3} \rightarrow \mathbb{R}^{3}$ which maps each point to an acceleration vector. 
We especially emphasize this representation of spatial information through field functions, because the modeling decision (or needs) of representing those functions in an explicit way or not, leads to specific constraints regarding the implementation of the environment.

\section{Four fundamental patterns}

We intend to show that a limited number of implementation families can be identified for MABS environments, depending on the nature of the metric space $(E, d)$, and on the choice of reifying (or not) cont $t_{t}$ Our point here is not to aim at a normative approach, nor at a comprehensive listing of cases, but rather at describing recurrent typical usages and their distinctive features. We introduce below the four fundamental patterns we have identified, with their principles, advantages and drawbacks. We show how to handle efficiently the search for neighbors and the motion of agents, i.e. how to actually compute the values of the functions $\nu_{t}^{r}$ and $\operatorname{pos}_{t+1}$ for each agent (resp. neighbors $(\mathrm{a}, \mathrm{r}$ ) and move_to $(a, p))$. Finally, we indicate when each pattern is best suited.

\subsection{The "AgentSet" pattern}

Principle. Each agent is endowed with an attribute pos containing $\operatorname{pos}_{t}(a)$. The environment is reduced to a set of agents $\left(\mathcal{A}_{t}\right)$ and the distance function $d$.

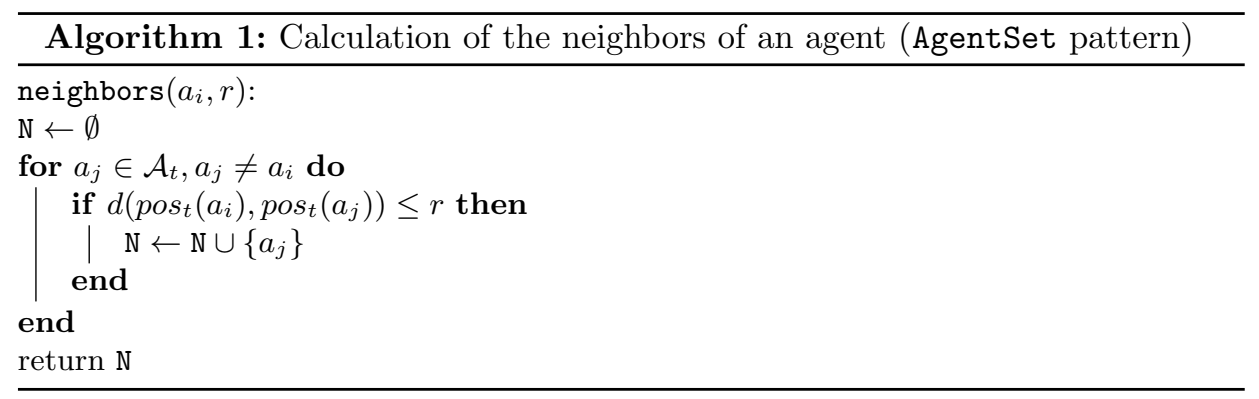

Advantages and drawbacks. Only the function $\operatorname{pos}_{t}$ is explicitly reified. Conversely, the computational complexity of neighbor perception in the simulation is in $\mathcal{O}\left(n^{2}\right)$, where $n=\left|\mathcal{A}_{t}\right|$. Indeed, each agent has to check its distance to the $(n-1)$ other agents (algorithm 1$)$. However, when an agent moves, it only has to modify its pos attribute as follows: move_to $\left(a_{i}, p\right): \operatorname{pos}_{t+1}\left(a_{i}\right) \leftarrow p$.

Usages. The AgentSet pattern proves convenient for implementing plain environments containing a small number of agents, otherwise finding the neighbors is costly. It is also suited for fields given in intension, since the corresponding functions have just to be implemented together with the set of agents and the distance function. 


\subsection{The "StandardGrid" pattern}

Principle. In many models, the environment to build is a discrete space organized as a geometric lattice, indexable by a system of discrete coordinates of dimension $k$ (such as $E=\mathbb{Z}^{k}$ ). The discrete positions can also be seen as "cells". To implementation such environments, the structure of the latter pattern is endowed with a grid, i.e. a tessellation of the environment, through a $k$-dimension array containing sets of agents. This explicit reification of function cont $t_{t}$ provides a direct access to all agents situated on any point (or "cell") $p$. Besides, all points adjacent to $p$ are retrieved easily, either by their coordinates or by cross-references between cells: hence, an immediate calculation of the neighbor points of $p$ (neighborhood in algorithm 2).
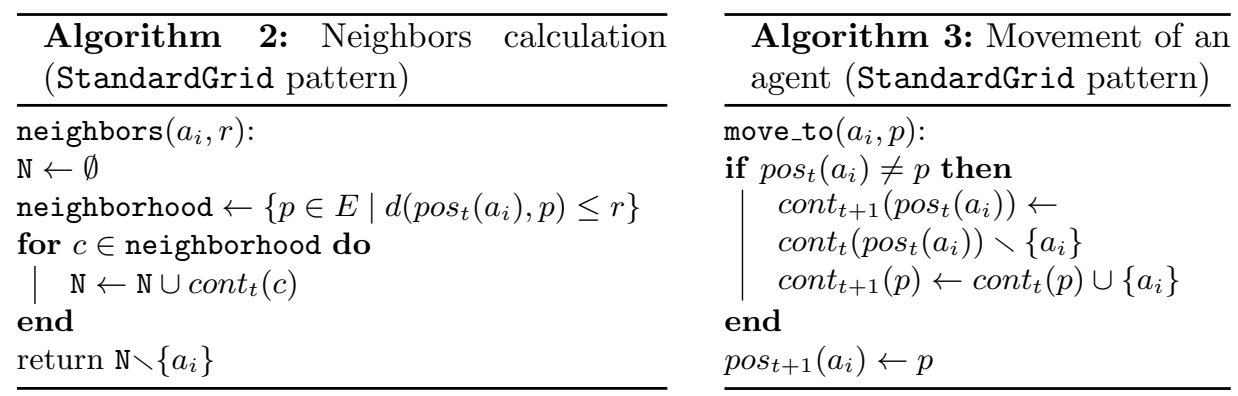

Advantages and drawbacks. Direct access to all agents on a point provides a considerable speed-up of neighbors search, since they are necessarily on the same cell as $a_{i}$ or on adjacent cells. Finding the neighbors of $n$ agents within a radius of $r$ (algorithm 2), has a cost in $\mathcal{O}\left(n . r^{k}\right)$ (e.g., with a Moore neighborhood and $k=2$, neighbors are in a square of $(2 r+1)^{2}$ cells). Conversely, moving an agent requires more complicated updates than in the previous pattern, in order to ensure consistency between $\operatorname{pos}_{t+1}$ and cont $_{t+1}$ (algorithm 3).

Usages. This pattern fits environments based on integer coordinates, especially if the average perception radius $\bar{r}$ of the agents (within which they search their neighbors) fulfils $\bar{r}^{k} \ll n$. It is also well suited to represent field functions even when given in extension (yet, assuming that those fields are defined on discrete coordinates): the values of the field on each point are held by a $k$-dimension array as well. This pattern also handles obstacles easily, when represented either by agents (which prevent the access to their cell), or by fields, or by the distance function (topology of the cells). Noteworthy, this grid-based approach is obviously not limited to square tessellations; many simulations e.g. in geography use hexagons [16], which also constitute a lattice of $\mathbb{R}^{2}$. We can also mention a very frequent simplification of this pattern: when the model assumes that two agents cannot occupy the same position, then the grid is simply a $k$-dimension array of agents (instead of a set of agents). 


\subsection{The "AggregateGrid" pattern}

Principle. The previous pattern cannot be used when the environment is based on a continuous space, because of the practical impossibility to provide exhaustive adjacency relations. Yet, if the function cont $t_{t}$ cannot be reified as such, a discrete approximation can be build by "projecting" the continuous positions in $E$ on a discrete space $\mathbb{E} \subset E$. Therefore, a discretization of $E$, depending on a mesh size $m$, must be defined: cell $_{m}: E \rightarrow \mathbb{E}$. In other words we need: $\forall c \in \mathbb{E}, \exists c^{\prime} \in \mathbb{E}, c^{\prime} \neq c$ such that $d\left(c, c^{\prime}\right) \leq m$. For instance, taking $E=\mathbb{R}^{2}$, it is quite natural to choose $\mathbb{E}=\mathbb{Z}^{2}$; then a simple discretization function would be: $\forall(x, y) \in \mathbb{R}^{2}$, cell $^{m}(x, y)=\left(\left\lfloor\frac{x}{m}\right\rfloor,\left\lfloor\frac{y}{m}\right\rfloor\right.$ ) (where $\lfloor u\rfloor$ denotes the floor of $u$ ). An "aggregate" form of the function cont $t_{t}$ can then be defined, so as to calculate the set of all agents situated in a "cell" $c: \forall c \in \mathbb{E}$, cell_cont $_{t}^{m}(c)=\left\{a \in \mathcal{A}_{t} \mid\right.$ cell $\left.^{m}\left(\operatorname{pos}_{t}(a)\right)=c\right\}$.

Now, the function cell_cont $t_{t}^{m}$ can be reified exactly like in the previous pattern, through a $k$-dimension array cell_cont which contains sets of agents (the set of all agents situated in cell $\left.\mathrm{cell}_{m}(p)\right)$.
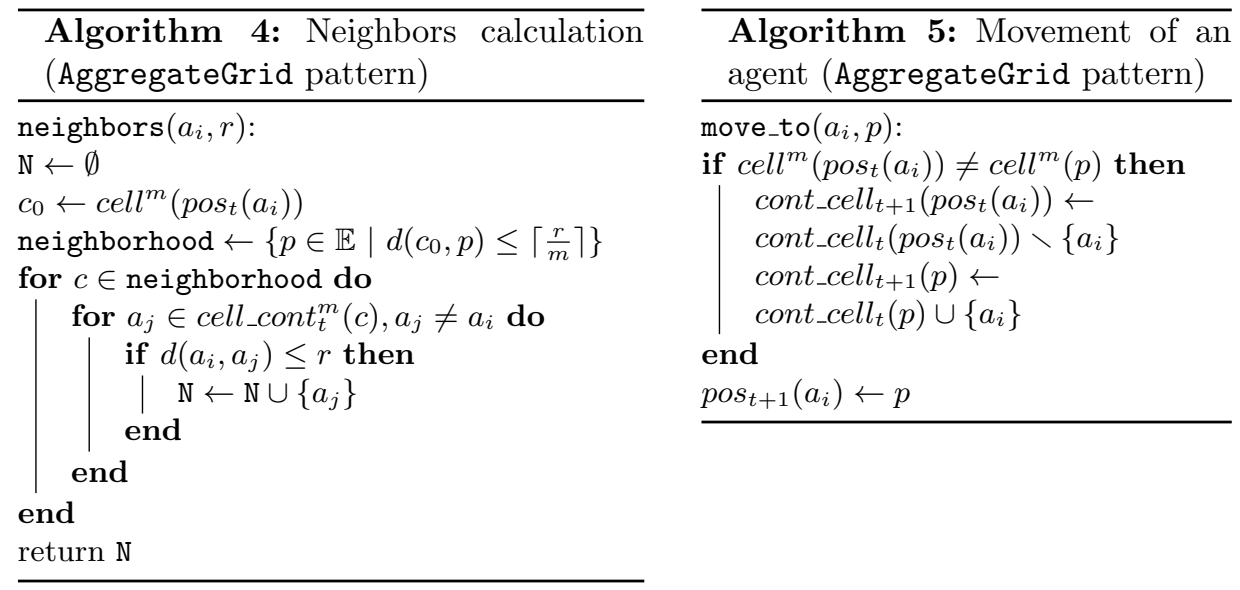

Advantages and drawbacks. Due to the discretization of the continuous space, this pattern benefits from the computational speed-up of a grid structure (cf. algorithm 4). At the same time, the arbitrary size of the mesh $m$ allows a fine tuning of this discretization if needed: the computational cost of neighbors detection for $n$ agents, within a radius of $r$, is in $\mathcal{O}\left(n \cdot\left(\left\lceil\frac{r}{m}\right\rceil\right)^{k} \cdot q\right)$ (where $\lceil u\rceil$ denotes the ceiling of $u$ and $q$ the density of agents i.e. the average number of agents in each cell). Again, the counterpart is a more complicated technique for updating the content of cells when agents move (cf. algorithm 5).

Usages. This pattern is relevant for modeling continuous environments containing a large number of agents. It can be also useful for implementing a discrete environment where the "natural" mesh size of 1 would be quite inefficient (i.e. 
when $\bar{r} \gg 1$ ). Like in the StandardGrid pattern, the representation of fields is quite straightforward, provided that the mesh size $m$ leads to a realistic approximation w.r.t. the spatial scale of the field. This approach can be extended to other tessellation methods (e.g. hexagons in the plane), including partition methods suited for heterogeneous spatial distribution of agents (e.g. Voronoi, quadtrees, etc.): the key principle is that each cell must have direct access to adjacent cells.

\subsection{The "SocialNet" pattern}

Principle. Each agent owns an attribute acquaintances which contains the set of all agents it knows and can interact with. Then pos $_{t}$ is bijective, i.e. the key issue is not knowing the places where the agents are situated, but rather the accessibility relations between places. Here, the topology of the environment comes first, because acquaintances relations are equivalent to the adjacency matrix of a graph (possibly directed if some acquaintances are not reciprocal): $\forall i, j, e_{i j}=1$ if $a_{j} \in$ acquaintances $_{t}\left(a_{i}\right), 0$ otherwise.

Thus, two very different situations may occur. If agents are allowed to interact with their own acquaintances only, the neighbors $\left(a_{i}, r\right)$ function is defined only for $r=1$, and trivially returns the acquaintances attribute. The environment can be considered "virtual" (or "purely communicating" [1]), being distributed through the acquaintances lists. On the contrary, if agents are allowed to interact within a wider range, then the cost of the corresponding breadth-first search of "neighbors" within a radius of $r$, is in $\mathcal{O}\left(\bar{q}^{r}\right)$ (with $\bar{q}$ the average size of acquaintances lists). Instead, the distance function can be reified through a distance matrix $\left(\delta_{i j}\right)$ where $\forall i, j \delta_{i j}=d\left(\operatorname{pos}_{t}\left(a_{i}\right), \operatorname{pos}_{t}\left(a_{j}\right)\right)$. It gives the shortest paths in the graph (computed using e.g. the Floyd-Warshall algorithm [17]). Then, the determination of the neighbors follows the same method as in the AgentSet pattern (algorithm 1).

Advantages and drawbacks. The trivial case is quite simple to implement. In the second situation, using a distance matrix provides the ability for any agent to interact with neighbors within an arbitrary radius. Yet, if changes occur in the acquaintances relations, this matrix has to be updated by recomputing the shortest paths, which is in $\mathcal{O}\left(n^{3}\right)$ in the Floyd-Warshall algorithm.

Usages. This pattern is naturally suited to "social" environments though it actually only assumes topological hypotheses regarding the accessibility relations between agents, regarded as nodes of a graph. Obviously, the approach can be extended with few transformations in situations where acquaintances are associated with weights.

\section{Combining patterns}

The pattern-based approach to environments we defend here, is rather a continuation of the method sketched in [9], than an attempt to build a unique, complex, 
monolithic first-class abstraction which is able to deal with all concerns at the same time as proposed in [18]. Without prejudging the interest of such integrated models for the analysis, design or implementation of MAS, we prefer to follow an orthogonal approach which ensures on the contrary a clear separation of concerns (in the same sense as in software engineering). Therefore we argue that it is more advisable to combine several of those fundamental patterns.

For instance, in a case where simulated robots have to explore a building, they are indeed situated in a continuous environment where they find obstacles (other entities), and at the same time may exchange information with their peers (the network of their acquaintances). Thus, "the" environment is actually a combination between the "AggregateGrid" and the "SocialNet" patterns. The neighbors of a robot is composed of, on the one hand, the entities perceived in the 3D surrounding space, and on the other hand, other robots that can be reached through radio communications.

Noteworthy, a multi-agent based simulation platform like NetLogo [19] actually provides an implementation for each of the patterns we propose, although not explicitly identified as such. The "AgentSet" is a native data type in the NetLogo language, which contains the collection of all agents of a kind. The "StandardGrid" pattern is a built-in feature due to the existence of NetLogo patches, which are $1 \times 1$ square cells discretizing the environment. The "AggregateGrid" pattern is realized by the fact that each NetLogo "turtle", which is endowed with floating-point coordinates, is located on a single patch (hence patches implement the two patterns simultaneously). The "SocialNet" pattern is provided by the ability to build links between turtles (and use them to detect "link-neighbors"). Besides, those NetLogo concepts do not imply any special use either as physical or social features, and are meant to be used together for implementing the various needs of a simulation model.

Thus, we note that our pattern-based approach leads to a unification of concepts of physical and social environments, that are usually kept distinct. Besides, it also pushes the designer to decompose "the" environment of the MAS, which is usually seen as a rather complex whole, into as many atomic environments as needed, each one with a univocal purpose and implementation.

\section{Conclusion and perspectives}

In this paper, we assume two main purposes for an "atomic environment": the spatial or social placement of agents, and the provision of information that cannot be represented by agents at the chosen modeling scale. Thus, an atomic environment is defined primarily as a space, endowed with a distance measure, with positioning and content functions, and aimed at finding agents and neighbors of agents: those features lead to a limited number of design patterns. The four patterns presented here seem to cover a wide range of applications in agentbased simulation.Besides, instead of modeling a single, complex environment in a MAS, we recommend to rather combine several patterns with a clear separation of concerns and purposes. 


\section{References}

1. Ferber, J.: Multi-Agent Systems. An Introduction to Distributed Artificial Intelligence. Addison Wesley (1999)

2. Russell, S., Norvig, P.: Artificial Intelligence. Prentice Hall (1995)

3. Okuyama, F., Bordini, R., da Rocha Costa, A.: ELMS: An environment description language for multi-agent simulation. In Weyns, D., et al., eds.: E4MAS'04. Volume 3374 of LNCS. Springer (2005) 91-108

4. Weyns, D., Parunak, H., Michel, F., Holvoet, T., Ferber, J.: Environments for multiagent systems. State-of-the-Art and research challenges. In Weyns, D., et al., eds.: E4MAS'04. Volume 3374 of LNCS. Springer (2005) 1-47

5. Ricci, A., Viroli, M., Omicini, A.: CArtAgO: A framework for prototyping artifactbased environments in MAS. In Weyns, D., et al., eds.: E4MAS'06. Volume 4389 of LNCS. Springer (2007) 67-86

6. Behrens, T.M., Hindriks, K.V., Dix, J.: Towards an environment interface standard for agent platforms. Ann. Math. Artif. Intell. 61(4) (April 2011) 261-295

7. Helleboogh, A., Vizzari, G., Uhrmacher, A., Michel, F.: Modeling dynamic environments in multi-agent simulation. J. Auton. Agents and Multi-Agent Systems (JAAMAS) 14(1) (2007) 87-116

8. Weyns, D., Holvoet, T.: A reference architecture for situated multiagent systems. In Weyns, D., et al., eds.: E4MAS'06. Volume 4389 of LNCS. Springer (2007) 1-40

9. Schelfthout, K., Coninx, T., Helleboogh, A., Holvoet, T., Steegmans, E., Steegmans, E., Weyns, D.: Agent implementation patterns. In: Workshop on AgentOriented Methodologies, 17th Annual ACM Conf. on Object-Oriented Programming, Systems, Languages, and Applications (OOPSLA). (2002) 119-130

10. Gamma, E., Helm, R., Johnson, R., Vlissides, J.: Design Patterns, Elements of Reusable Object-Oriented Software. Addison Wesley (1994)

11. Kubera, Y., Mathieu, P., Picault, S.: Everything can be agent! In: 9th Int. Joint Conf. on Auton. Agents and Multi-Agent Systems (AAMAS). (2010) 1547-1548

12. Picault, S., Mathieu, P.: An interaction-oriented model for multi-scale simulation. In: 22nd Int. Joint Conf. on Artificial Intelligence (IJCAI), AAAI (2011) 332-337

13. Latombe, J.C.: Robot Motion Planning. Kluwer Academic Publishers (1991)

14. Van Dyke Parunak, H.: Between agents and mean fields. In Villatoro, D., et al., eds.: MABS XII. Volume 7124 of LNCS. Springer (2011)

15. Colorni, A., Dorigo, M., Maniezzo, V.: Distributed optimization by ant colonies. In: 1st European Conf. on Artifical Life (ECAL), Elsevier (1991) 134-142

16. Sanders, L., Pumain, D., Mathian, H., Guérin-Pace, F., Bura, S.: SIMPOP: a multi-agents system for the study of urbanism. Environment and Planning B 24 (1997) 287-305

17. Floyd, R.: Algorithm 97: Shortest path. Communications of the ACM 5(6) (1962) 345

18. Weyns, D., Omicini, A., Odell, J.: Environment as a first class abstraction in multiagent systems. J. Auton. Agents and Multi-Agent Systems (JAAMAS) 14(1) (2007) 5-30

19. Wilensky, U.: Netlogo. Center for Connected Learning and Computer-Based Modeling, Northwestern University, Evanston, IL (1999) http://ccl.northwestern.edu/netlogo/. 